\title{
AMENDMENTS
}

\section{Author Correction: The origin of galaxy colour bimodality in the scatter of the stellar-to-halo mass relation}

Weiguang Cui iD, Romeel Davé (iD, John A. Peacock, Daniel Anglés-Alcázar and Xiaohu Yang

Correction to: Nature Astronomy https://doi.org/10.1038/s41550-021-01404-1, published online 5 July 2021.

In the version of this Article originally published, Weiguang Cui was missing the affiliation "Department of Astronomy, School of Physics and Astronomy, Shanghai Jiao Tong University, Shanghai, China"; it has now been added and the affiliations renumbered accordingly. All versions of the Article have been amended.

Published online: 28 July 2021

https://doi.org/10.1038/s41550-021-01456-3

(c) The Author(s), under exclusive licence to Springer Nature Limited 2021 